\title{
Nutritional screening of elderly patients - A health improvement approach to practice
}

\author{
K. Farrer, D. Melia, B. Blackett, H. Lloyd, C. Beadman, E. Donaldson and S. Lal \\ Salford Royal NHS Foundation Trust (SRFT), Stott Lane, Salford, M6 8HD, UK
}

\begin{abstract}
Malnutrition is both a common and concerning problem with almost $60 \%$ of elderly care in-patients being at risk ${ }^{(1)}$. It is well established that malnourished patients will have poorer clinical outcomes ${ }^{(2)}$ and that malnutrition is a key factor in prolonging length of stay in elderly patients ${ }^{(1)}$. The Malnutrition Universal Screening Tool (MUST) has been developed as a method of identifying these at-risk patients and has been advocated across the NHS since $2003^{(3)}$.

The nutrition team at SRFT were keen to understand local current practice and then take action to improve the situation. In order to achieve this the team designed a quality improvement project based on the Model for Improvement ${ }^{(4)}$. 3 elderly care wards were invited to participate in the first phase of this project as these wards have many patients at high risk of malnutrition. Each developed a multidisciplinary improvement team of ward staff to work on improving MUST screening.

The nutrition team designed a driver diagram which articulated the aim of the project and the key workstreams that would be necessary to achieve it. This was used as a clear visual method of communicating the project to the ward teams. Each month the timeliness (within 6 hours of admission) and accuracy (compared to a dietician assessment) of MUST scores on the three wards was reviewed. Ward teams received monthly feedback of their results to create ownership, maintain enthusiasm and generate momentum for improvement. Baseline data identified that a MUST screen was performed in $<60 \%$ of patients and in those with an assessment only $65 \%$ were accurate.

Plan-Do-Study-Act (PDSA) cycles were used to rapidly test changes in the ward areas. Tests included a nutrition study day, one-to-one ward based nutrition training, focus on the use of alternative anthropometric measurements, development of a training pack and identification of the challenges to undertaking accurate and timely assessments.

During the project all the wards achieved an improvement in the proportion of patients screened with one ward achieving $100 \%$. It was identified that strong ward leadership was a key factor in this ward's success. There was also some evidence that the drive to screen within 6 hours resulted in less accurate assessments and that assessments within 24 hours might be more accurate. We also identified that only $60-70 \%$ patients screened had a nutrition care plan and thus that screening does not necessarily result in action to improve nutritional state.
\end{abstract}

The nutrition team is now working with wards to evaluate the link between timeliness and accuracy of assessment in more detail. We are also looking at the role of Health Care Assistants in MUST screening. The team continues to work on improving nutritional screening but is now focussed on developing reliable processes, using quality improvement methodologies and the application of reliability science, to ensure the delivery of appropriate interventions to patients identified as high risk.

1. Stratton et al. (2006) Br J Nutr 95, 325-330.

2. NICE (2006) CG32.

3. Elia M (2003) BAPEN

4. Langley et al. (2009) The Improvement Guide ( $2^{\text {nd }}$ Edn) P. 24. 